\title{
Rare Etiology of Vascular Bands on Esophageal Manometry
}

\author{
Nishant Tripathi ${ }^{1}$, Lisabeth A Selby ${ }^{2}$, Bahaaeldeen S. Ismail ${ }^{2}$ \\ 1) University of Kentucky, Department of Hospital Medicine, Lexington; 2) University of Kentucky, Department of Internal Medicine, Division \\ of Digestive Diseases and Nutrition, Lexington, USA
}
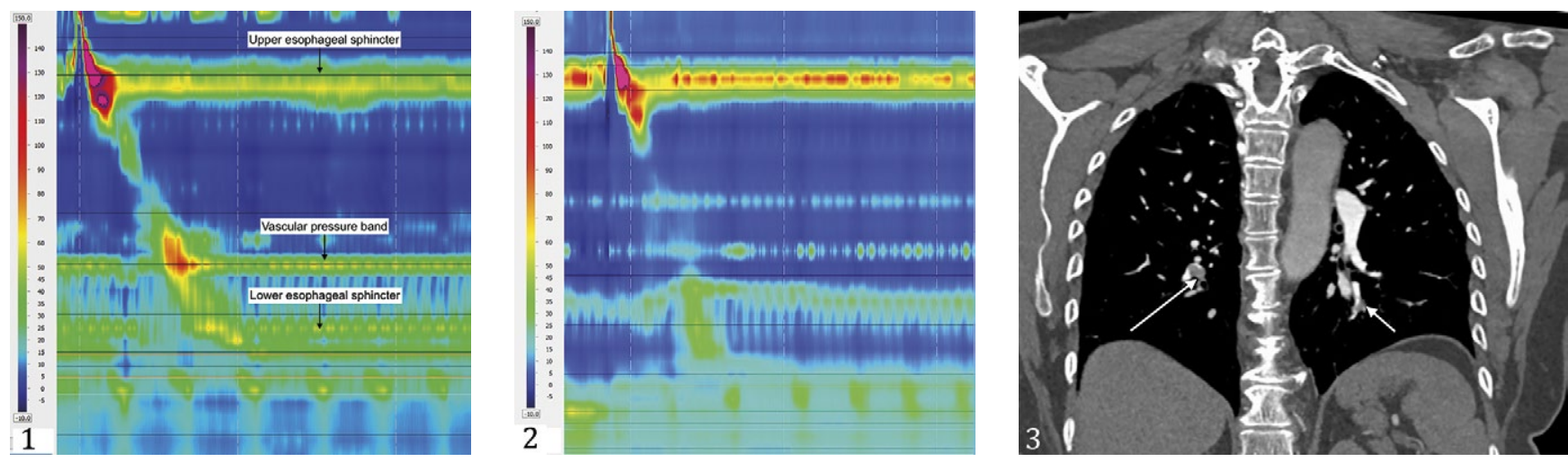

A 68-year-old female with a history of pulmonary embolism (PE), hiatal hernia and regurgitation was referred for highresolution manometry (HRM) prior to the hiatal hernia repair. During the procedure, she experienced acute dyspnea and leftsided chest pain that persisted after the study. HRM revealed hypotensive lower esophageal sphincter with hiatal hernia, weak peristalsis and prominent vascular bands (Fig. 1) that were not present on the previous manometry two years ago (Fig. 2). Urgent chest computed tomography (CT) angiography revealed emboli in the branches of the right and left pulmonary arteries (Fig. 3, arrows), along with right heart strain as evidenced by increased right ventricular diameter. Moreover, CT also identified abnormal courses of the esophagus and ectatic aorta. Apixaban was initiated and she eventually underwent laparoscopic hiatal hernia repair with Toupet fundoplication.

Vascular bands, also termed pulsatile pressure zones, vascular pressure bands, or horizontal pressure bands, identified during HRM occur frequently secondary to esophageal compression with abnormal vasculature, or cardiomegaly [1]. Previous studies identified that up to $56 \%$ of the HRM studies detected vascular bands [1-3]; moreover, the aortic arch, the aberrant subclavian artery and left atrium were categorized as the most implicated cardiovascular structures resulting in pressure bands [2]. While the clinical significance of vascular bands remains uncertain, they have been postulated to cause dysphagia in patients with additional underlying risk factors, such as, advanced age, atherosclerosis, and valvular heart disease [2]. Our patient's subtle vascular anomaly had not previously affected her esophagus. Nonetheless, the hemodynamic changes resulting from PE likely lead to unmasking of this anomaly. The resultant esophageal compression between the strained main pulmonary artery and the tortuous ectatic aorta [2] possibly instigated the interval change in her HRM. Esophageal compression due to acute vascular changes is an area for further research and should be considered in the appropriate clinical scenario [3].

Corresponding author: Nishant Tripathi, nishant55@icloud.com

Conflicts of interest: None to declare.

\section{REFERENCES}

1. Lis S, Vachhani H, Tanner S, et al. Vascular pressure bands on highresolution esophageal manometry with impedance studies. Dis Esophagus 2020;33:doz093. doi:10.1093/dote/doz093

2. Chaudhry NA, Zahid K, Keihanian S, Dai Y, Zhang Q. Transmitted cardiovascular pulsations on high resolution esophageal impedance manometry, and their significance in dysphagia. World J Gastroenterol 2017;23:7840-7848. doi:10.3748/wjg.v23.i44.7840

3. Stagias JG, Ciarolla D, Campo S, Burrell MI, Traube M. Vascular compression of the esophagus: a manometric and radiologic study. Dig Dis Sci 1994;39:782-786. doi:10.1007/BF02087424 